protective role of Hsp90 not only elevates bcl-2/bax and bcl-xL/bax expression but also decrease cleaved-caspase3 expression via downregulating TLR-4 and ErbB2 membrane receptors. By binding to TLR-4 and ErbB2, Hsp90 activates the PI3K/Akt and ERK1/2 pathways. Hsp90 also down regulates the pro-apoptotic protein bax. It is demonstrated that exogenous Hsp90 elevates the expression levels of bcl-2/bax and bcl-xL/bax by activating the TLR-4 and ErB2 downstream PI3K/Akt and ERK1/2 pathways, which decreases cleaved caspase-3.

Conclusion Hsp90 significantly protects MSCs against apoptosis induced by hypoxia and serum deprivation. These findings demonstrates a novel and effective treatment strategy against MSC apoptosis in cell transplantation.

\section{e0202 EFFECTS OF RANOLAZINE ON ACTION POTENTIAL AND CONTRACTION FORCE IN GUINEA PIG PAPILLARY MUSCLES}

doi:10.1136/hrt.2010.208967.202

Guotao Wang, Ping Yang. Department of Cardiology, China-Japan Union Hospital of Jilin University, Changchun, China

Objective To observe the effects of ranolazine on the action potential and contraction force in guinea pig papillary muscles. To explore the mechanism of ranolazine anti-arrhythmia and myocardial ischaemia.

Methods 18 healthy adult guinea-pigs were randomly divided into $\mathrm{H}_{2} \mathrm{O}_{2}(200 \mathrm{mmol} / \mathrm{l})$ groups, ranolazine $(10 \mathrm{mmol} / \mathrm{l})+\mathrm{H}_{2} \mathrm{O}_{2}$ groups and TTX $(2 \mathrm{mmol} / \mathrm{l})+\mathrm{H}_{2} \mathrm{O}_{2}$ groups, with six guinea pigs in each group compared before and after administration to observe the effects of ranolazine on the papillary muscles.

Results $\mathrm{H}_{2} \mathrm{O}_{2}$ could increase action potential durations measured at $50 \%$ repolar... moreization levels and $90 \%$ repolarisation levels were prolonged $(p<0.001)$. There was reduced myocardial contractility $(p<0.05)$ in contraction force in the guinea pigs compared to before administration. Ranolazine can inhibit action potential durations measured at the $50 \%$ repolar... moreization levels and the $90 \%$ repolarisation levels were prolonged by $\mathrm{H}_{2} \mathrm{O}_{2}$, but the effect was weaker compared to that of TTX. Ranolazine and TTX could improve myocardial contractile force by reducing the $\mathrm{H}_{2} \mathrm{O}_{2}$-induced. Conclusion ranolazine could reduce action potential duration the $\mathrm{H}_{2} \mathrm{O}_{2}$-induced and increase contraction force. TTX performs a similar role.

\section{e0203 ATHEROSCLEROSIS IMPAIRS CXCR4 EXPRESSION AND FUNCTION IN BONE MARROW CELLS}

doi:10.1136/hrt.2010.208967.203

Xu Qiyuan, Yu Hong. Second Affiliated Hospital, Zhejiang University College of Medicine, Hangzhou, China

Objectives The chemokine stromal cell-derived factor-1 (SDF-1) plays a critical role in mobilising CXCR4-positive precursor cells in the bone marrow and may be essential for efficient vascular regeneration and repair. We recently reported that CXCR4 and the angiogenic potential of bone marrow derived cells (BMCs) were regulated positively by calcium and negatively by ageing. We hypothesise that atherosclerosis may also affect CXCR4 surface expression and functions. This study is to determine that atherosclerosis defects BMCs both on the decreased population of CXCR4 + progenitor cells and on the impaired mobility as well as to explore possible mechanisms.

Methods We established atherosclerosis model on ApoE-/- mice under at least 6-month High Fat Diet (group Ath), and used 3 4week aged ApoE-/- mice as control (group Ctrl). In order to evaluate impact of the disease on CXCR4 expression from gene transcription to protein synthesis, surface CXCR 4 expression on BMCs from the two groups of mice were analysed with FCM; the mRNA of CXCR4 was detected by real-time PCR. To compare the inducibility of CXCR4 expression in response to environmental change, $1 \mathrm{mM}$ $\mathrm{CaCl}_{2}$ was applied as an extracellular stimuli to treat BMCs for $4 \mathrm{~h}$. To examine how $\mathrm{BMC}_{\text {Ath }}$ are defect on calcium-induced CXCR4 surface expression, calcium influx was analysed by measuring the increase of intracellular calcium after BMCs were mixed with $\mathrm{CaCl}_{2}$. Cell-cell adhesion assays were used to detect the SDF-1 mediated adhesion of BMCs to the HUVEC monolayer and vertical invasion assays were used respectively to quantify trans-endothelial migration of BMCs in response to a gradient of SDF-1.

Results $\mathrm{BMC}_{\mathrm{Ath}}$ have less number of cells expressing CXCR4 as compared to $\mathrm{BMC}_{\mathrm{Ctrl}}(8.54 \pm 1.96 \%$ vs $13.75 \pm 3.94 \% ; \mathrm{n}=10, \mathrm{p}<0.01)$ CXCR4 expression on $\mathrm{BMC}_{\mathrm{Ctrl}}$ could be enhanced by calcium, but CXCR4 surface expression in $\mathrm{BMC}_{\mathrm{Ath}}$ increased significantly lesser then $\mathrm{BMC}_{\mathrm{Ctrl}}(11.24 \pm 1.31 \%$ vs $26.59 \pm 4.92 \% ; \mathrm{n}=10, \mathrm{p}<0.01)$. It is partly because of the defective calcium influx in $\mathrm{BMC}_{\text {Ath }}$ which reduced the CXCR4 gene transcription, consequently leading to impaired responses on calcium-induced CXCR4 surface expression. $\mathrm{BMC}_{\text {Ath }}$ showed weaker lower mobility and lower trans-endothelial migration ( $0.80 \pm 0.11 \mathrm{~mm}$ vs $1.17 \pm 0.15 \mathrm{~mm} ; \mathrm{n}=4, \mathrm{p}<0.05)$, and this was not enhanced by calcium pretreatment.

Conclusions Atherosclerosis impairs CXCR4 surface expression on BMCs and related cell functions.

\section{e0204 HEAT SHOCK PROTEIN 90 ENHANCES RAT MESENCHYMAL STEM CELLS MIGRATION VIA PI3KAKT AND ERK12 PATHWAYS}

doi:10.1136/hrt.2010.208967.204

Gao Feng, Hu Xinyang, Xie Xiaojie, Xu Qiyuan, Wang Yaping, Liu Xianbao, Wu Rongrong, Xiang Meixiang, Sun Yong, Wang Jian-an. Second Affiliated Hospital, Zhejiang University College of Medicine, Hangzhou, China

Objective Heat shock protein 90 (HSP90) is a chaperone for several client proteins involved in transcriptional regulation, signal transduction, and cell cycle control. HSP90 is abundantly expressed by a variety of tumour types and has been recently targeted for cancer therapy. The objective of this study is to determine the role of Hsp90 in regulating the migration of Mesenchymal stem cells and to determine the mechanism. We hypothesised that inhibition of Hsp90 impairs the MSCs migration via PI3K/Akt and ERK1/2 signalling pathways.

Methods The MSCs were cultured from femoral and tibia. The ability for MSCs cells to migrate is to be determined by the wound healing assay and transwell assay. The activity of matrix metalloproteinase-2 (MMP-2) and matrix metalloproteinase-9 (MMP-9) were estimated by gelatin -zymography. The mRNA levels of MMP-2, MMP-9, CXCR4 and VCAM-1 were detected by real-time PCR. The protein expression of MMP-2, MMP-9 and ERK1/2, phospho-ERK1/2, Akt and phospho-Akt were determined by Western-blot

Results Treatment with RhHsp90 $\alpha$ significantly enhances MSCs migration from $9.83 \pm 2.48$ to $48.65 \pm 2.81$ cells. Treatment with sirhsp $90 \alpha$ significantly decreased MSCs migration compared with treatment of hsp90 $\alpha$ from $63.33 \pm 9.61$ to $13.00 \pm 4.38$ cells. Pretreat with 17-AAG, wortmannin, U0126, decreased MSCs migration to $13.33 \pm 1.29,15.33 \pm 2.1,16.5 \pm 3.3$ cells, respectively. Treatment with RhHsp90 $\alpha$ enhanced the MSCs secretion of MMP-2 and MMP-9, as well as significantly increasing the activity of MMP-9, and increasing the expression of CXCR4 and VCAM-1. PI3K/Akt and ERK signalling pathways mediate the promotion of MSCs migration by RhHsp90a. 
Conclusions Our data showed that Hsp90 promotes MSCs migration, elevate the expression of MMP-2, MMP-9, CXCR4 and VCAM1. PI3K/Akt and ERK signalling pathways mediates these effects. Hsp90 is a candidate drug for enhancement of MSCs migration.

\section{e0205 MICRORNA REGULATION OF CARDIOMYOCYTE LINEAGES FROM BONE MARROWDERIVED MESENCHYMAL STEM CELLS}

doi:10.1136/hrt.2010.208967.205

Wu Rongrong, Mo Xiaozhou, Hu Xinyang, Wang Yaping, Xiang Chunsheng, Wang Jian-an. Second Affiliated Hospital, Zhejiang University College of Medicine, Hangzhou, China

Objective ischaemic heart disease is the leading cause of morbidity and mortality all over the world. Cardiomyocytes from bone marrow-derived mesenchymal stem cells (MSCs) offer great potential for repairment of the infracted heart. However, this approach has been limited by inefficient differentiation of MSCs into cardiomyocytes. To overcome such a problem, the underlying regulation mechanisms for cardiac differentiation should be elucidated. MicroRNAs (miRNAs) are small noncoding RNAs of $\sim 23$ nucleotides that control post-transcriptional gene expression. Recently, miRNA has been widely shown to regulate key cellular events such as cell proliferation, cell differentiation. The purpose of this study is to determine the role of miRNAs during cardiac differentiation from MSCs.

Method Firstly, we established a model of cardiac differentiation from rat bone marrow-derived MSCs using $10 \mu \mathrm{M}$ 5-Aza, and performed a global miRNA analysis using EXIOON miRNA array to identify characteristic miRNA at different stage of differentiation. After being validated by real-time qRT-PCR and target gene prediction, several miRNAs such as miRNA-145 were further chosen to reveal its function during cardiac differentiation.

Results miRNA profiling revealed that miR-145 expression increased during cardiac differentiation, especially at 12 days of treatment (2.25-fold change vs untreated MSCs). Compared to other tissues such as liver, brain, kidney, miRNA-145 expression is highest in rat heart tissue. Gain-of-function methods using pre-miR-145 showed the enchancement of cardiac differentiation, confirmed by immunocytochemical staining with cardiac-specific myosin heavy chain antibody.

Conclusion miR-145 may be a critical regulator for cardiomyocyte lineage in our 5-Aza-induced differentiation system.

\section{e0206 BONE MARROW MESENCHYMAL STEM CELL MIGRATION DEPENDS IN PART ON KV21 CHANNEL ACTIVITY}

doi:10.1136/hrt.2010.208967.206

Hu Xinyang, Yu Shanping, Wang Jian-an, Wei Ling. Second Affiliated Hospital, Zhejiang University College of Medicine, Hangzhou, China

Objective The present study was designed to examine 1 . whether hypoxic preconditioning (HP) increases the migration potential of mesenchymal stem cells (MSC) and 2. the mechanistic basis for this effect.

Methods MSCs derived from green fluorescent protein (GFP)transgenic mice were cultured under either normoxia (N-MSC) or hypoxia $\left(0.5 \% \mathrm{O}_{2}\right)$ (HP-MSC) for $24 \mathrm{~h}$. Wound healing and transwell assays were performed to assess cell migration in vitro. Potassium channel expression and FAK phosphorylation were analysed by western blot analysis, whole cell patch clamp recording was used to measure delayed rectifier $\mathrm{K}^{+}$currents. Interactions between the Kv2.1 potassium channel and FAK were analysed via immunoprecipitation. N-MSC or HP-MSC were intravenously injected via tail vein in myocardial rat, and migration of MSC was assessed in vivo.

Results Both wound healing and transwell assays showed that, compared with controls, shRNA-mediated knock-down of the Kv2.1 potassium channel significantly reduced the ability of $\mathrm{N}$ MSC to migrate. While exposure to hypoxia enhanced MSC migration both in vitro and in vivo, this effect was inhibited in presence of either tetra-ethylammonium (TEA) or elevated extracellular potassium. The potential effects of hypoxia exposure on MSC migration were significantly reduced by Kv2.1 knockdown. Hypoxic pre-conditioning significantly increased the expression of Kv2.1. Similarly, compared to normoxic controls, delayed rectifier $\mathrm{K}^{+}$currents in HP-MSC were much greater. Lastly, HP-mediated increases in phosphorylation of FAK ${ }^{576 / 577}$ and FAK $^{397}$ were neutralised by either addition of TEA or elevation of extra-cellular potassium levels.

Conclusion These findings demonstrate that KV2.1 plays an important role in the regulation of MSC migration. HP enhances the ability of MSC to migrate both in vitro and in vivo, and this effect may be mediated, at least in part, through activation of Kv2.1 potassium channels and the FAK pathway.

\section{e0207 RB1 REVERSES $\mathbf{H}_{\mathbf{2}} \mathbf{0}_{\mathbf{2}}$ INDUCED SENESCENCE IN HUMAN UMBILICAL ENDOTHELIAL CELLS VIA MODULATING ENOS PATHWAY}

doi:10.1136/hrt.2010.208967.207

Liu Dinghui, Qian Xiaoxian. The Third Affiliated Hospital of Sun Yat-sen University, Guangzhou, China

Objective Cellular senescence of endothelial cells has been proposed for its involvement in endothelial dysfunction and atherogenesis. This study investigates the effects of ginsenoside Rb1, a major constituent of ginseng, on $\mathrm{H}_{2} \mathrm{O}_{2}$-induced endothelial senescence and molecular changes in primary human umbilical vein endothelial cells.

Methods Prematurely senescent human umbilical vein endothelial cells (HUVECs) were induced by treatment with $\mathrm{H}_{2} \mathrm{O}_{2}$ as judged by senescence-associated $\beta$-galactosidase assay (SA- $\beta$-gal), cell morphological appearance, and plasminogen activator inhibitor-1 (PAI-1. expression. Total nitric oxide (NO) production was measured using Griess reaction. Endothelial NOS (eNOS), PAI RNA expressions were analysed by real time PCR. Total eNOs, pS1177 eNOS and pT495 eNOS protein expressions were analysed by westernblotting.

Results Treatment with $40 \sim 100 \mu \mathrm{M} \mathrm{H}_{2} \mathrm{O}_{2}$ caused $26.8 \sim 63.8 \%$ of the cells to be SA- $\beta$-gal positive. Pretreated with Rb1 markedly inhibited SA- $\beta$-gal activity dose-dependently. Also, Rb1 can reduce the expression of PAI which was increased in the $\mathrm{H}_{2} \mathrm{O}_{2}$ treated group. In $\mathrm{H}_{2} \mathrm{O}_{2}$ treated groups, eNOS mRNA expression decreased, while $\mathrm{Rb} 1$ can effectively restore its mRNA expression. eNOS activity of HUVECs was inhibited by decreasing eNOS phosphorylation at Ser-1177 and increasing eNOS phosphorylation at Thr-495 in $\mathrm{H}_{2} \mathrm{O}_{2}$ treated groups. While in $\mathrm{Rb} 1$ pretreated groups, the both exhibited opposite changes. Consistent with these findings, $\mathrm{Rb} 1$ does in fact increase NO levels. All the inhibitory effects of Rb1 on senescence were completely obliterated by L-NAME, the NOS inhibitor.

Conclusion Rb1 can effectively protect HUVEC from senescence through modulating the expression of eNOS. 